\title{
Determinación de la fugacidad para el ciclohexano a las condiciones de la Ciudad de México mediante el uso de una ecuación de estado
}

DOI: $10.46932 / \mathrm{sfjdv2n2-214}$

Received in: March 1st, 2021

Accepted in: May 30th, 2021

\section{Juan Daniel López Melo}

Estudiante de noveno semestre de Ingeniería Química, Universidad Anáhuac México Norte. Universidad Anáhuac México Norte: Av. Universidad Anáhuac 46, Col. Lomas Anáhuac, Huixquilucan, Estado de México. C.P. 52786.

Correo: juandaniel.lopez05@gmail.com ; juan.melo@anahuac.mx

\section{Fernanda Atondo Hernández}

Estudiante de noveno semestre de Ingeniería Química, Universidad Anáhuac México Norte. Universidad Anáhuac México Norte: Av. Universidad Anáhuac 46, Col. Lomas Anáhuac, Huixquilucan, Estado de México. C.P. 52786. Correo: feratondo98@gmail.com; fernanda.atondohe@anahuac.mx

\section{Ximena Villegas Pañeda}

Doctora en Ingeniería Química, profesora de la Universidad Anáhuac México Norte. Universidad Anáhuac México Norte: Av. Universidad Anáhuac 46, Col. Lomas Anáhuac, Huixquilucan, Estado de México. C.P. 52786.

Correo: ximena.villegas@anahuac.mx

\section{Alma Delia Rojas Rodríguez}

Maestra en Ingeniería Química, profesora de la Universidad Anáhuac México Norte. Universidad Anáhuac México Norte: Av. Universidad Anáhuac 46, Col. Lomas Anáhuac, Huixquilucan, Estado de México. C.P. 52786.

Correo: alma.rojas@anahuac.mx

\section{RESUMEN}

La fugacidad es la medida del potencial químico para ajustar el valor de una presión. Está directamente relacionada con la tendencia de una sustancia para mantener la estabilidad de una fase (líquida, sólida, vapor o gas). A una temperatura y presión fijas, una sustancia específica tendrá una fugacidad diferente para cada fase. Aquella fase con la menor fugacidad será la más estable.

Palabras clave: fugacidad, ecuaciones de estado, presión de vapor, coeficiente de fugacidad, propiedades críticas.

\section{INTRODUCCIÓN}

Para el cálculo de la fugacidad de una sustancia, se optó por calcular la fugacidad del ciclohexano, teniendo los datos teóricos de presión crítica, temperatura crítica y factor acéntrico de esta sustancia y considerando la presión del sistema, como la presión atmosférica del lugar en donde se realizó el experimento, que fue en la Ciudad de México. Cabe destacar que se hizo el cálculo de la fugacidad sin 
necesidad de usar un simulador, los cuales requieren un costo para su uso y no están disponibles para el público en general. Para el cálculo se uso únicamente una hoja de cálculo y ecuaciones de estado.

\section{OBJETIVO}

Determinar los valores de fugacidades mediante la medición de temperaturas de ebullición y cálculo de presiones de vapor del ciclohexano a la presión atmosférica de la ciudad de México.

\section{MARCO TEÓRICO}

La fugacidad es una propiedad termodinámica auxiliar que actúa como factor de corrección para transformar una ecuación ideal, en un caso real, con dimensiones de presión. Es una medida de la tendencia que tiene una sustancia a escapar de la fase en la que se encuentra por influencias de factores externos. El coeficiente de fugacidad es la relación que existe entre la fugacidad y la presión del componente como gas ideal, este factor se considera como la corrección del estado ideal, sus valores oscilan entre 0 y 1 .

La ecuación de estado tipo virial es una de las ecuaciones de estado de gases reales que posee una mayor flexibilidad a la hora de ajustar los datos experimentales y está basadas en desarrollos en serie de tipo polinómico. Al igual que con otras ecuaciones de estado, las de tipo virial surgen de un modelo mecánico estadístico que contempla las fuerzas intermoleculares. A medida que la densidad aumenta a temperatura constante, los choques binarios entre las moléculas se producen con mayor frecuencia, el segundo coeficiente del virial, que da cuenta de esos choques binarios, no será nulo y esto hace que el cociente $\frac{p V_{m}}{R T}$ aumente, lo cual indica un predominio de las fuerzas de repulsión que hace que el volumen ocupado por el gas sea mayor que el del gas ideal. El segundo coeficiente del virial B, tiene gran importancia pues puede medirse con facilidad y su conocimiento permite estudiar los gases reales a bajas y moderadas presiones, pues los restantes términos de los desarrollos en serie son despreciables.

Las propiedades críticas son el conjunto de condiciones físicas de presión, temperatura y volumen, a las cuales la densidad y otras propiedades del líquido y gas se vuelven idénticas. Estas propiedades son únicas (una sola presión, una sola temperatura). Los volúmenes molares de líquidos se pueden determinar por medio de ecuaciones cúbicas, pero sus resultados no son muy exactos. Las ecuaciones generalizadas permiten obtener el cálculo de volúmenes molares de líquidos saturados. La ecuación de Racket sirve para determinar el volumen del líquido de cierta sustancia. A partir del cálculo de las propiedades anteriores y del uso de las ecuaciones de estado se pueden calcular los parámetros para determinar la fugacidad de una sustancia. 


\section{METODOLOGÍA}

1. Se colocó en un vaso de precipitados $5 \mathrm{~mL}$ de ciclohexano
2. En un vaso de precipitados, se colocaron $150 \mathrm{~mL}$ de aceite de cocina
3. En el tubo de ensaye con ciclohexano, se introdujo un tubo capilar cerrado

5. Se midió la temperatura, colocando un termopar dentro del tubo de ensaye con la muestra y el capilar

de cocina

4. Se colocó el tubo de ensaye (con la muestra de ciclohexano y el capilar) dentro del vaso de precipitados con aceite en una
6 . En el momento en el que se vió el burbujeo (ebullición), en forma de un rosario, porque el burbujeo se da repetidas veces, se registró la temperatura de ebullición
7. Al obtener la temperatura de ebullición, se regresaron los $5 \mathrm{~mL}$ de ciclohexano a su contenedor original, pues no hubo reacción química
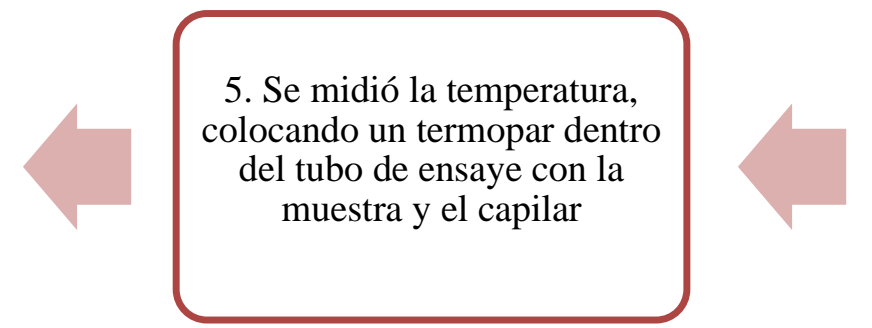
parrilla previamente nrecalentada

8. Se realizaron los cálculos y el análisis necesario para determinar si la presión de saturación era mayor o menor que la del medio (presión atmosférica de la Ciudad de México) para determinar si se está hablando de un líquido o un gas, para poder emplear las fórmulas correctamente para el cálculo de la fugacidad.
9. Obtención del gráfico fugacidad vs temperatura 
Figura 1. Obtención de la temperatura de ebullición del ciclohexano

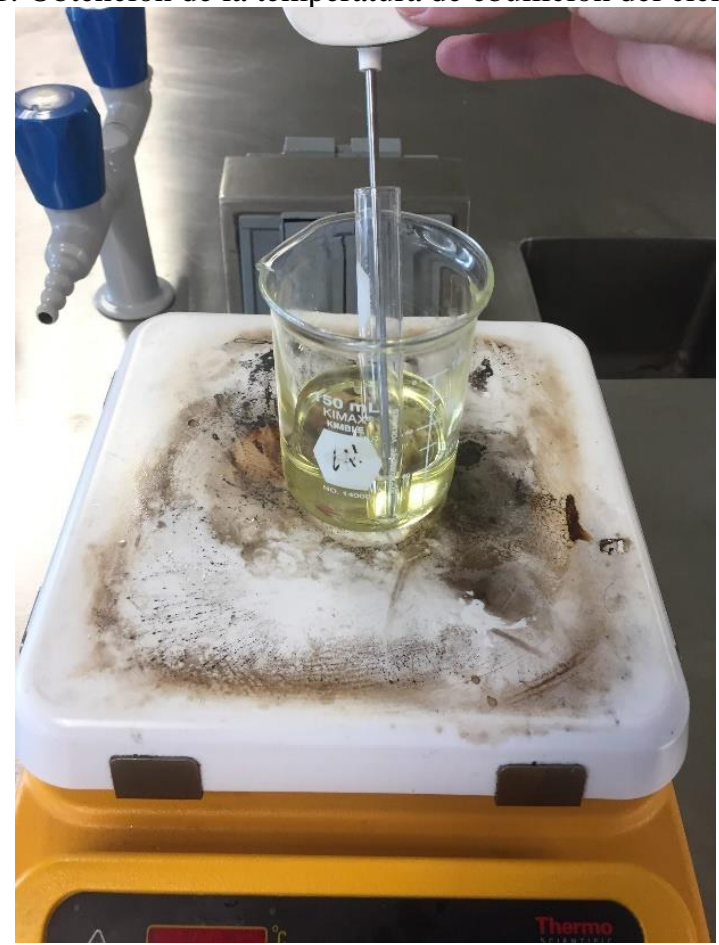

\section{RESULTADOS}

Datos:

Se empleó la ecuación de Antoine para calcular la presión de saturación del ciclohexano con la temperatura de ebullición registrada experimentalmente.

Tabla 1. Constantes de Antoine del ciclohexano

\begin{tabular}{|c|c|c|c|}
\hline & A & B & C \\
\hline Ciclohexano & 6.8413 & 1201.531 & 222.647 \\
\hline
\end{tabular}

Temperatura de ebullición del ciclohexano: $71^{\circ} \mathrm{C}\left(71^{\circ} \mathrm{C}+273.15\right)=344.15 \mathrm{~K}$

Presión del sistema (presión en la ciudad de México): $78.127 \mathrm{kPa}$

Constante universal de los gases: $8.314 \mathrm{~kJ} / \mathrm{kmolK}$

Datos del ciclohexano obtenidos del libro Smith van Ness:

$\omega=0.210$

Temperatura crítica: $553.6 \mathrm{~K}$

Presión crítica: 40.73 bar(100kPa/1bar $)=4073 \mathrm{kPa}$

Como la presión de saturación obtenida, es menor que la presión del sistema, se considera que se habla de un líquido, por lo que se manejará la presión de saturación en los cálculos.

Datos adicionales por estar manejando un líquido, obtenidos del libro Smith van Ness: 


$$
\begin{gathered}
\mathrm{Vc}=308 \frac{\mathrm{cm}^{3}}{\mathrm{~mol}} \\
\mathrm{Zc}=0.273
\end{gathered}
$$

1. Ecuación para calcular la temperatura reducida

$$
T_{r}=\frac{T}{T c}=\frac{344.15 \mathrm{~K}}{553.6 \mathrm{~K}}=0.622
$$

2. Ecuación virial truncada en el segundo coeficiente

$$
\begin{gathered}
B^{0}=0.083-\frac{0.422}{T_{r}^{1.6}}=0.083-\left(\frac{0.422}{0.622^{1.6}}\right)=-0.8199 \\
B^{1}=0.139-\frac{0.172}{T_{r}^{4.2}}=0.139-\left(\frac{0.172}{0.622^{4.2}}\right)=-1.1275 \\
B=\left(\frac{R T_{c}}{P_{c}}\right)\left(B^{0}+\omega B^{1}\right) \\
B=\left(\frac{8.314 \frac{k J}{k m o l ~ K} \times 553.6 K}{4073 k P a}\right) \times(-0.8199+(0.210 \times-1.1275) \\
B=-1.1941 \frac{\mathrm{m}^{3}}{\mathrm{kmol}^{2}}
\end{gathered}
$$

3. Ecuación logarítmica despejada para calcular el coeficiente de fugacidad

$$
\begin{gathered}
\ln \ln \varnothing=\frac{B P}{R T} \\
\ln \ln \varnothing=\frac{-1.1941 \frac{\mathrm{m}^{3}}{\mathrm{kmol}} \times 74.895 \mathrm{kPa}}{8.314 \frac{\mathrm{kJ}}{\mathrm{kmol} \mathrm{K}} \times 344.15 \mathrm{~K}}
\end{gathered}
$$

$$
\ln \ln \emptyset=-0.0313
$$




$$
\begin{gathered}
\emptyset=\exp (\ln \ln \emptyset) \\
\emptyset=\exp (-0.0313) \\
\varnothing=0.9692
\end{gathered}
$$

4. Ecuación de Poyntig para líquidos para el cálculo de la fugacidad:

$$
\begin{gathered}
f=\emptyset^{\text {sat }} P^{\text {sat }} \exp \exp \left(\frac{V}{R T}\left(P-P^{\text {sat }}\right)\right) \\
f=(0.9692 \times 74.895 \mathrm{kPa}) \times \exp \exp \left(\frac{0.115 \frac{\mathrm{m}^{3}}{\mathrm{kmol}}}{8.314 \frac{\mathrm{kJ}}{\mathrm{kmol} \mathrm{K}} \times 344.15 \mathrm{~K}} \times(78.127 \mathrm{kPa}-74.895 \mathrm{kPa})\right) \\
f=72.599 \mathrm{kPa}
\end{gathered}
$$

5. Ecuación de Racket para volúmenes de líquidos:

$$
\begin{gathered}
V^{l}=V_{c} Z_{c}{ }^{\left(1-T_{r}\right)^{2 / 7}} \\
V^{l}=308 \frac{\mathrm{cm}^{3}}{\mathrm{~mol}} \times\left(0.273^{(1-0.622)^{\frac{2}{7}}}\right. \\
V^{l}=115.1944 \frac{\mathrm{cm}^{3}}{\mathrm{~mol}} \times\left(\frac{1000 \mathrm{~mol}}{10^{6} \mathrm{~cm}^{3}}\right) \times\left(\frac{1 \mathrm{~m}^{3}}{1 \mathrm{kmol}}\right) \\
V^{l}=0.115 \frac{\mathrm{m}^{3}}{\mathrm{kmol}}
\end{gathered}
$$

Tabla 2. Tabla para la obtención de la gráfica fugacidad-temperatura

\begin{tabular}{|c|c|}
\hline Temperatura (K) & Fugacidad (kPa) \\
\hline 344.15 & 72.59 \\
\hline 350 & 72.63 \\
\hline 360 & 72.69 \\
\hline 370 & 72.75 \\
\hline 380 & 72.81 \\
\hline 390 & 72.86 \\
\hline
\end{tabular}


Figura 2. Gráfica Fugacidad vs Temperatura

FUGACIDAD-TEMPERATURA

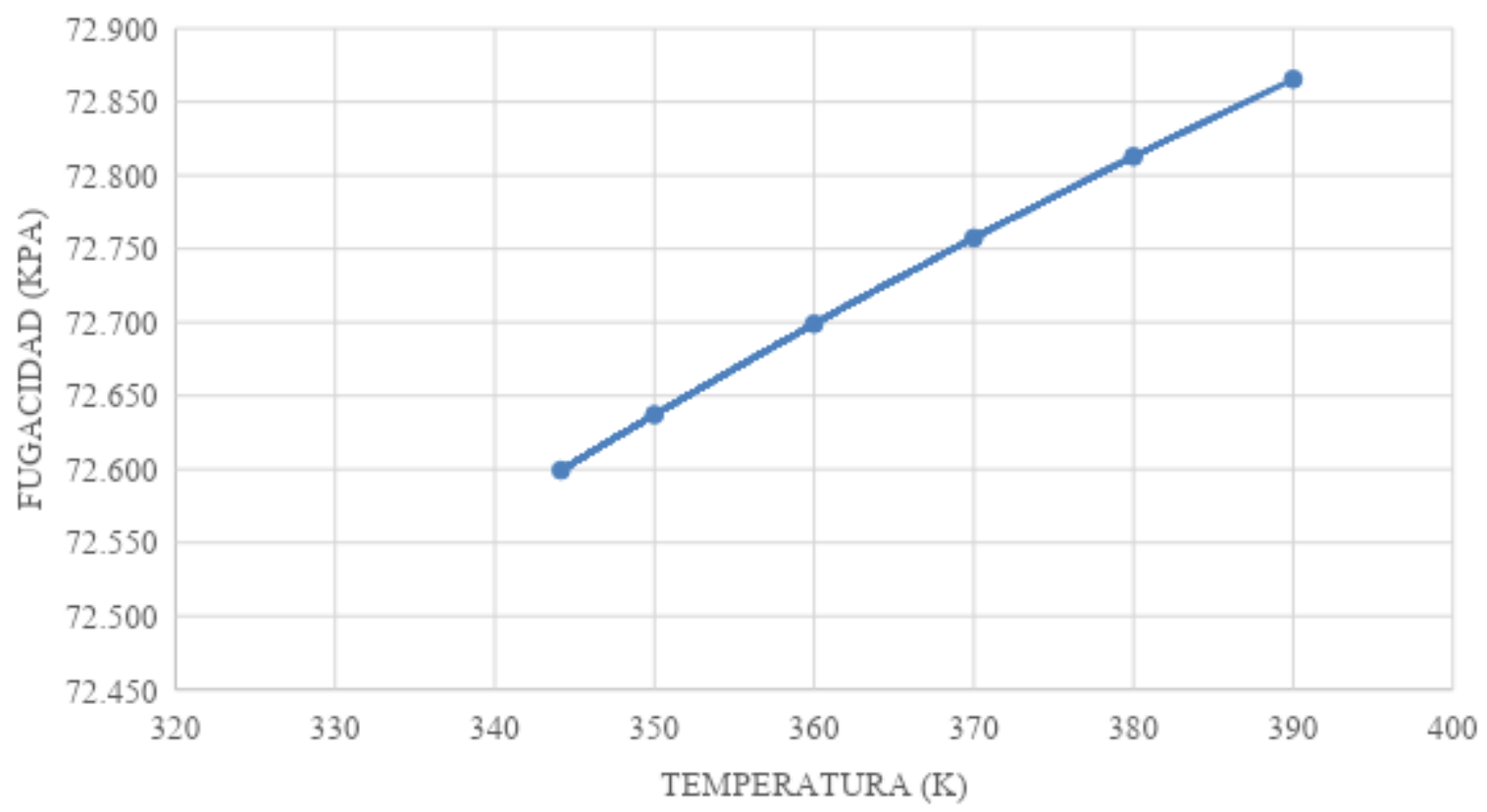

\section{ANÁLISIS DE RESULTADOS}

Para el cálculo de la fugacidad, se empleó el ciclohexano, usando solamente una muestra de $5 \mathrm{~mL}$, en donde la presión total del sistema es de $78.12 \mathrm{kPa}$. Se calculó la presión de saturación del ciclohexano con la ecuación de Antoine a la temperatura de $71^{\circ} \mathrm{C}$, para obtener una presión de saturación de $74.89 \mathrm{kPa}$. Como la presión de saturación del ciclohexano fue menor que la presión del sistema, se considera que se encuentra en fase líquida, por lo tanto se usa para los cálculos la presión de saturación. La fugacidad es una corrección de la idealidad, al calcular esta propiedad termodinámica, se puede comparar un caso ideal, con un caso real. Esta propiedad es la tendencia de una sustancia para salir de la fase en la que se encuentra, bajo las influencias de las condiciones de presión y temperatura. La fugacidad de un líquido es distinta a la fugacidad de un sólido, para los datos obtenidos se tuvo que hacer una corrección con la ecuación de Poynting, pues se debe tener en cuenta la diferencia entre la presión de vapor del componente y la presión total del sistema.

Las determinaciones de la fugacidad se hicieron en una hoja de cálculo sin la necesidad de usar algún simulador, los cuáles no son de fácil acceso y son caros, por lo tanto en este trabajo se explica como se puede obtener la fugacidad sin la necesidad de recurrir a estos programas. 


\section{CONCLUSIONES}

Los valores de la fugacidad dependen del valor de la presión atmosférica del lugar, pues, dependiendo de las diferencias entre presiones de vapor y presiones del sistema, podrá darse la fugacidad en líquido o en vapor.

Como se mencionó con anterioridad, la relevancia de calcular la fugacidad en una hoja de cálculo, es la facilidad para el público y principalmente para estudiantes que no cuentan con el acceso a simuladores o programas para obtener dichos valores y poder facilitar proyectos o trabajos con fines académicos.

\section{AGRADECIMIENTOS}

Agradecemos al Doctor Víctor Hugo del Valle Muñoz, por permitirnos el uso del laboratorio de Química Orgánica para el desarrollo de este proyecto, así como a la Dirección de Investigación de la Universidad Anáhuac México por el patrocinio para participar en el $54^{\circ}$ Congreso Mexicano de Química, $38^{\circ}$ Congreso Nacional de Educación Química y Expoquímica 2019 de la Sociedad Química de México. 


\section{REFERENCIAS}

Atkins, P. W., Fisicoquímica, 3ª Edición, USA, Addison-Wesley Iberoamericana, 1991.

Cárdenas, J. Fugacidad y Coeficiente de Fugacidad. Recuperado el: 28/06/2021. Disponible en: https://www.academia.edu/15031084/FUGACIDAD_Y_COEFICIENTE_DE_FUGACIDAD

Castellan, G. W., Fisicoquímica, 2ª Edición, USA, Addison-Wesley Iberoamericana, 1987.

Chang, R. (2002). QUÍMICA, séptima edición. Colombia: McGraw-Hill Companies, Inc.

Kopp, M. (2019) Fugacidad, Coeficiente de fugacidad y método Gamma/Phi para el equilibrio vaporlíquido en mezclas. Recuperado el: 28/06/2021. Disponible en: https://www.researchgate.net/publication/334284806_Fugacidad_Coeficiente_de_fugacidad_y_metodo_ GammaPhi_para_el_equilibro_vapor_-_liquido_en_mezclas

Labarta, J.A. (2007) Fugacidad; Coeficiente de fugacidad; Corrección de Poynting. Recuperado el: 28/06/2021. Disponible en: http://rua.ua.es/dspace/handle/10045/13321\#vpreview

López, J. y Atondo, F. (2019, octubre) Determinación de la fugacidad para el ciclohexano a las condiciones de la Ciudad de México mediante el uso de una ecuación de estado. Comunicación presentada en el 54 ${ }^{\circ}$ Congreso Mexicano de Química, $38^{\circ}$ Congreso Nacional de Educación Química y Expoquímica, Puebla, México.

Smith, Van Ness, Abbott (1997) Introducción a la termodinámica en ingeniería química. (5ª edición). México: McGraw Hill.

Universidad de Valladolid. Ecuaciones de Estado. Recuperado 15 de junio del 2019, Sitio web: https://alojamientos.uva.es/guia_docente/uploads/2013/469/45750/1/Documento24.pdf 